\title{
PROTECTIVE EFFECT OF 6-SHOGAOL AGAINST ENDOTOXIN-INDUCED PERIODONTITIS IN RATS
}

\author{
HONG YAN QI ${ }^{1 *}$ and BING HAN ${ }^{2}$ \\ ${ }^{1}$ Department of Stomatology, First Hospital Affiliated to Lanzhou University, Gansu, China \\ ${ }^{2}$ Northwest University for Nationalities, China
}

\begin{abstract}
Periodontal diseases are the most prevalent bacterial ailments, affecting $10-15 \%$ of the global population, and eventually leading to tooth loss if left untreated. Shogaols obtained from ginger (Zingiber officinale) exhibit significant anti-inflammatory activity. However, the antibacterial potential of shogaols against periodontitis remains unexplored. Therefore, we investigated the effects of 6-shogaol (6-SH) on various factors responsible for periodontitis such as inflammation. Escherichia coli endotoxin was used to induce experimental periodontitis, and the effects of 6-SH on hydrogen peroxide, superoxide anion, myeloperoxidase activity, and lipid peroxides were estimated together with cathepsin B, cathepsin D, $\beta$-glucuronidase, acid phosphatase, and $\mathrm{C}$-reactive protein activity in serum. In addition, the levels of ascorbic acid, $\alpha$-tocopherol, ceruloplasmin, reduced glutathione, superoxide dismutase, catalase, glutathione peroxidase, and glutathione S-transferase were estimated in serum after 6-SH treatment. Reactive oxygen species and lipid peroxide levels were significantly reduced in the 6-SH-treated group. Moreover, lysosomal enzyme (cathepsin B, cathepsin D, $\beta$-glucuronidase and acid phosphatase) and acute-phase protein (C-reactive protein and fibrinogen) levels significantly declined after administration of 6-SH. Meanwhile, non-enzymatic antioxidant systems (e.g., ascorbic acid, $\alpha$-tocopherol, ceruloplasmin, and reduced glutathione) and antioxidant enzymes (e.g., catalase, superoxide dismutase, glutathione peroxidase, and glutathione S-transferase) were significantly increased in the 6-SH-treated group. These results suggest a protective effect of 6-SH against experimental periodontitis via the regulation of key disease markers.
\end{abstract}

Keywords: antioxidants, cathepsin B, cathepsin D, $\beta$-glucuronidase, periodontitis, 6-shogaol

The oral cavity is considered a paradise for the growth of microorganisms responsible for various infections and inflammatory disorders (1). Among diseases that cause oral infection, periodontal disease (PD) or periodontitis is defined as a bacterially induced disease of tooth-supporting (periodontal) tissues, and can eventually lead to tooth loss if left untreated. It is characterized by inflammation and bone loss and affects $10-15 \%$ of the global population (2). PD is caused by various gram-negative bacteria, such as Porphyromonas gingivalis, Prevotella intermedia, Actinobacillus spp., and Fusobacterium spp. (3). Moreover, the development of biofilms recalcitrant to treatment with current antibacterial agents exacerbates its progression (4).

Inflammation, together with oxidative stress, is a vital mediator of PD. Studies have shown that periodontitis induces excessive reactive oxidative species (ROS) production in periodontal tissue.
When periodontitis develops, ROS produced in the periodontal lesion diffuse into the bloodstream, causing the oxidation of blood molecules (i.e., circulating oxidative stress) including DNA, lipid, and protein oxidation, and inducing tissue damage. Such effects can be monitored based on the levels of $\alpha$ tocopherol, ceruloplasmin, reduced glutathione (GSH), superoxide dismutase (SOD), catalase (CAT), glutathione peroxidase (GPx), and glutathione S-transferase (GST). The host response to periodontal infection includes the production of several families of enzymes that are released by stromal, epithelial, or inflammatory cells. Studies of these enzymes in gingival crevicular fluid may help clarify the pathogenesis of PD and provide a rational basis for the development of novel therapeutics. The potential disease markers in gingival crevicular fluid included in this study can be broadly represented in two forms: biochemical mediators and

\footnotetext{
* Corresponding author: e-mail: hellenfoweinsa@yahoo.com
} 
products of inflammation (e.g., C-reactive proteins and fibrinogen), and host-derived enzymes (e.g., cathepsin B, cathepsin D, $\beta$-glucuronidase, and acid phosphatase). However, the therapeutic effectiveness of chemotherapeutic agents against PD is limited due to its multifactorial etiology. Therefore, there is an urgent need for innovative agents that can circumvent the problems faced by current regimens by acting on multiple pathways simultaneously.

Plants and plant-based drugs have been widely used as a source of medicinal agents for millennia (5). Approximately one-third of the top-selling drugs worldwide are natural products or derivatives because of their inherent unmatched chemical diversity and practically along with the possible absence of side effects (6-11). In this context, compounds isolated from ginger (Zingiber officinale Roscoe, Zingiberaceae) have shown potential positive effects on the treatment of many ailments. In particular, 6shogaol (6-SH), the dehydrated form of gingerol obtained from ginger, is a potent antioxidant, antiinflammatory, and antibacterial agent. Bacterial biofilms contain high concentrations of polysaccharides, proteins, nucleic acids, and lipids that are responsible for maintaining the structural integrity of the biofilm and providing an ideal matrix for bacterial growth. Recent studies suggest that Z. offici- nale inhibits the synthesis of exopolysaccharides and proteinaceous factors in coagulase-negative staphylococci strains and has antibiofilm activity (12-15). However, the efficacy of 6-SH against PD remains unknown. Therefore, in this study, we investigated the effects of 6-SH on various factors responsible for periodontitis to determine whether it has protective effects against PD.

\section{MATERIALS AND METHODS}

All synthetic substrates, including 6-SH, were obtained from Sigma Chemical Co. (USA ). For the animal experiment, male Wistar rats weighing approximately $250 \mathrm{~g}$ were procured from the central animal house of the institute, housed in polypropylene cages under strict veterinary supervision, and maintained in controlled rooms with a $12 \mathrm{~h}$ light/dark cycle. The animals received a standard laboratory diet and water ad libitum. The study was approved by the Institutional Animal Ethics Committee.

\section{Endotoxin preparation}

To produce the endotoxin required for the animal experiment, Escherichia coli was cultured on nutrient agar overnight to obtain the optimum
A

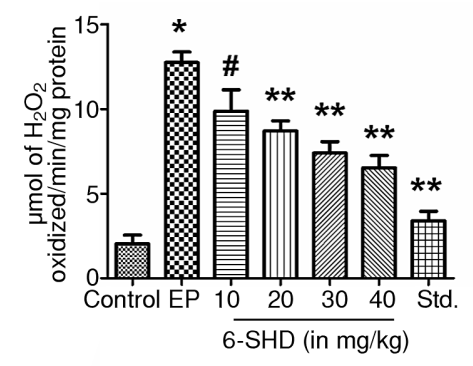

C

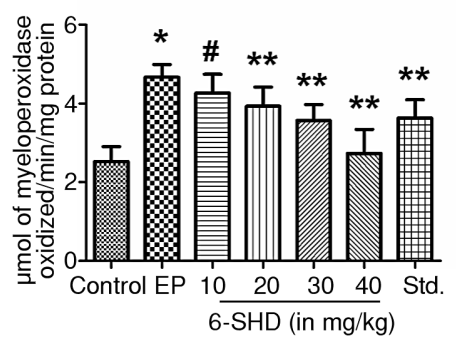

B

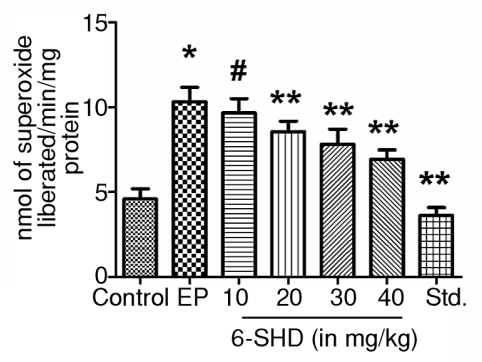

D

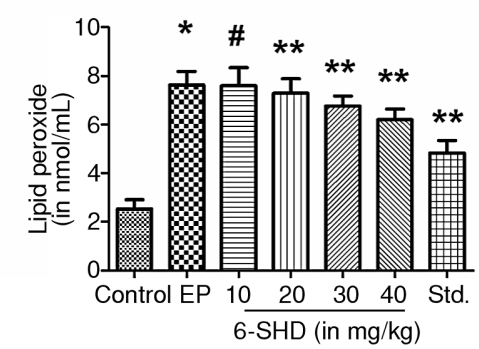

Figure 1. Effect of 6-SH on the levels of ROS and lipid peroxides in endotoxin induced experimental periodontitis. * $\mathrm{p}<0.01$ vs. control, ${ }^{*} \mathrm{p}<0.05,{ }^{* *} \mathrm{p}<0.01$ vs. EP group 


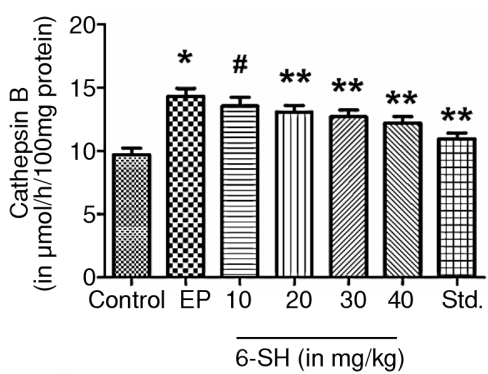

C

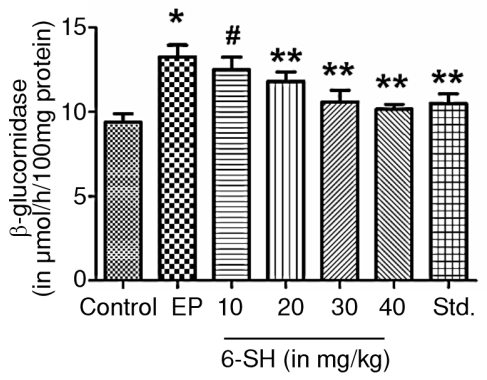

B

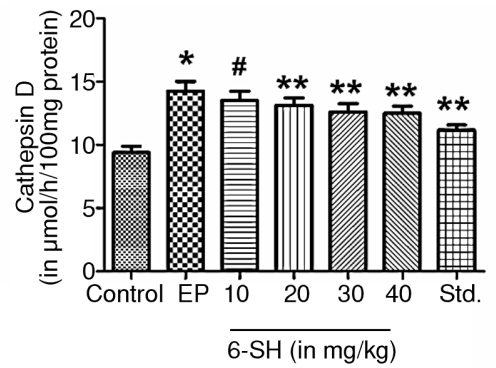

D

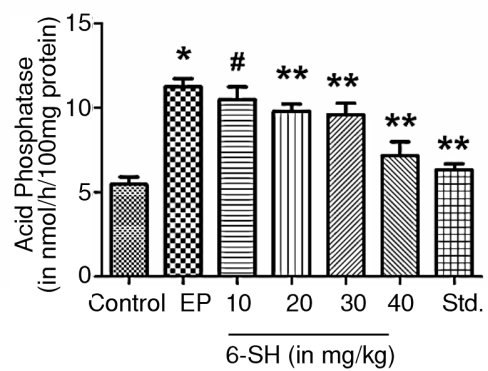

Figure 2. Effect of 6-SH on the levels of lysosomal enzymes in endotoxin induced experimental periodontitis. ${ }^{*} \mathrm{p}<0.01$ vs. control, $\mathrm{p} p<0.05,{ }^{* *} \mathrm{p}<0.01$ vs. EP group

colony count, which was subsequently washed with phosphate-buffered saline. The liquid collected after washing was thoroughly mixed with phenol in a separatory funnel. The aqueous phase was collected and extracted again with phenol, and this process was repeated three times. The resulting supernatant was precipitated with ethanol and sodium acetate. The precipitate containing endotoxin was readily isolated by centrifugation at $10000 \times g$ for $10 \mathrm{~min}$ and air-dried.

\section{Experimental periodontitis generation}

Experimental periodontitis (EP) was induced by administration of E. coli endotoxin. Rats received intragingival injection with either $10 \mu \mathrm{L}$ saline or 1 mg endotoxin dissolved in $1 \mathrm{~mL}$ saline into the labial and palatal aspects of the maxillary anterior gingivae and buccal to induce periodontitis. The rats were divided into the following treatments: control group, standard metronidazole-treated group (20 $\mathrm{mg} / \mathrm{kg}$ metronidazole), and 6-SH-treated groups (10, 20,30 , and $40 \mathrm{mg} / \mathrm{kg}$ 6-SH administered once per day in a 6-day experimental period via oral intubation). Each treatment group included six animals. At the end of the experimental period, animals were sacrificed by cervical decapitation under light ether anesthesia, and venous blood was collected. Bone and teeth of the right maxillary halves were dissected out and histopathological evaluation was performed on the maxillary halves.

\section{Estimation of biochemical parameters}

The effects of 6-SH as an antioxidant agent were quantified based on hydrogen peroxide $\left(\mathrm{H}_{2} \mathrm{O}_{2}\right)$, superoxide $\left(\mathrm{O}_{2}{ }^{\circ}\right)$, - myeloperoxidase (MPO) activity, and lipid peroxide (LPD) levels. In addition, the activity of cathepsin B, cathepsin D, $\beta$-glucuronidase, and acid phosphatase was determined using standard protocols. The activity of C-reactive protein in serum was estimated using the standard protocol. The fibrinogen assay was performed using a standard assay kit (Fibriquik ${ }^{\circledR}$ Organon Teknika, USA) according to the manufacturer's protocol. Ascorbic acid, $\alpha$-tocopherol, ceruloplasmin, GSH, SOD, CAT, GPx, and GST levels were estimated in serum, and the protein concentration in serum was determined using crystalline bovine serum albumin as a reference standard. 


\section{Histopathological analyses}

Tissues from the maxillary halves were dissected out, fixed in $10 \%$ buffered formalin solution for $24 \mathrm{~h}$, processed, and embedded in paraffin. Thin sections $(3 \mu \mathrm{m})$ were cut and stained with hematoxylin and eosin for analyses under light microscopy.

\section{Statistical analyses}

All data are presented as the means \pm standard deviations. The results were analyzed with one-way analysis of variance and the Bonferroni multiple range post hoc test using the statistical software GraphPad Prism ver. 5.0 (CA, USA). p values below 0.05 were considered statistically significant.

\section{RESULTS}

ROS are highly reactive and are produced continuously as by-products of cellular respiration as well as during enzymatic reactions. ROS comprise radical compounds (e.g., $\mathrm{O}_{2}^{\bullet},-$ hydroxyl radicals, and lipid hydroperoxides) and reactive nonradical compounds (e.g., singlet oxygen and $\mathrm{H}_{2} \mathrm{O}_{2}$ ). The levels of $\mathrm{H}_{2} \mathrm{O}_{2}, \mathrm{O}_{2}{ }^{\bullet},-$ MPO, and LPDs were aberrantly elevated in EP rats compared to the 6-SH- and standard-treated rats (Fig. 1). Meanwhile, ROS and LPD levels were significantly reduced in the 6-SHtreated group.

Figure 2 presents the effects of 6-SH on lysosomal enzymes including cathepsin B, cathepsin D, $\beta$-glucuronidase, and acid phosphatase. In humans, cathepsin B is encoded by the CTSB gene, belongs to a family of lysosomal cysteine proteases, and has an important role in intracellular proteolysis. Cathepsin D is a peptidase belonging to the family of aspartic peptidases. Its best-described function is intracellular catabolism in lysosomal compartments, although other physiological effects include hormone and antigen processing. $\beta$-glucuronidase catalyzes the hydrolysis of glucuronides and is

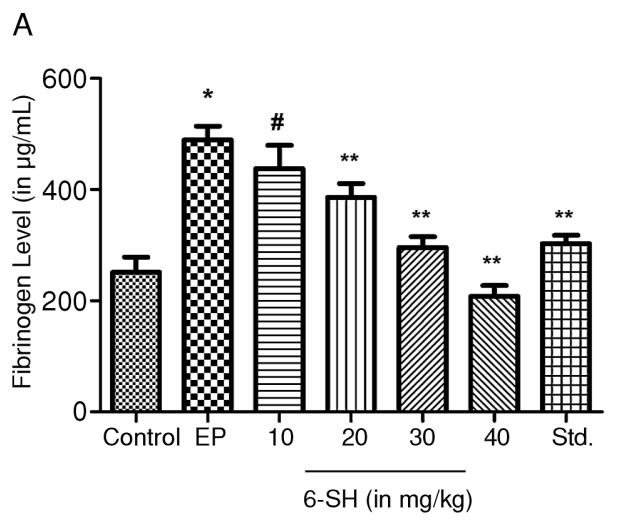

B

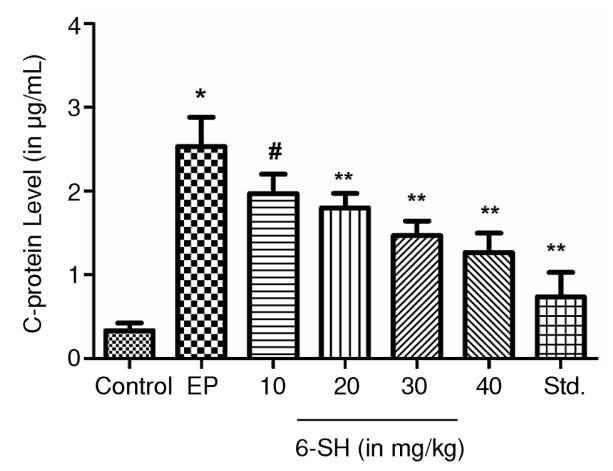

Figure 3. Effect of 6-SH on the levels of acute phase proteins in endotoxin induced experimental periodontitis. ${ }^{*} \mathrm{p}<0.01 \mathrm{vs}$. control, ${ }^{*} \mathrm{p}<0.05,{ }^{* *} \mathrm{p}<0.01$ vs. EP group 
A

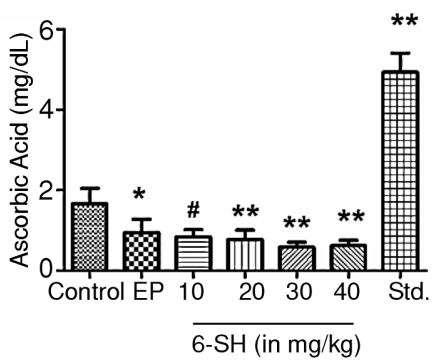

C

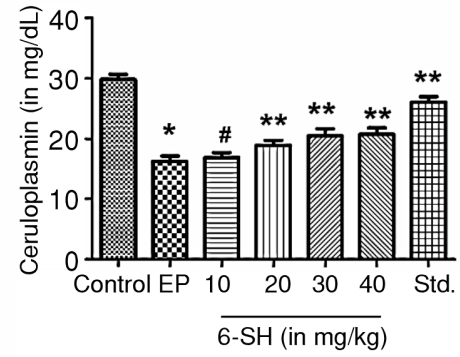

E

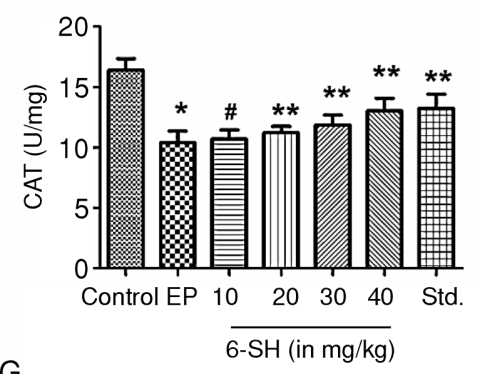

G

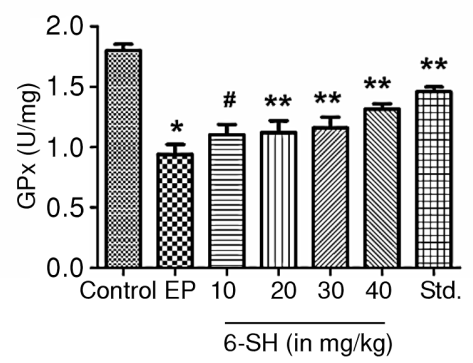

B

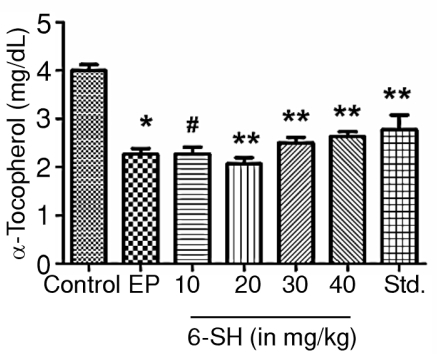

D

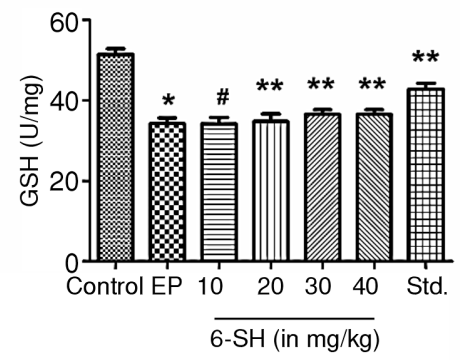

F

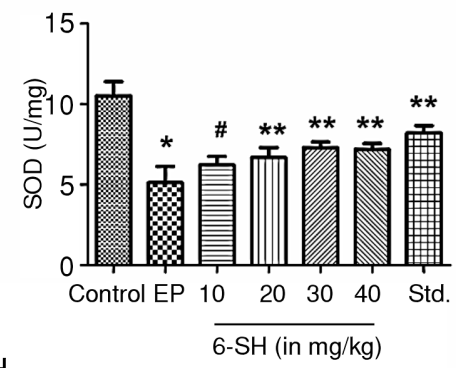

$\mathrm{H}$

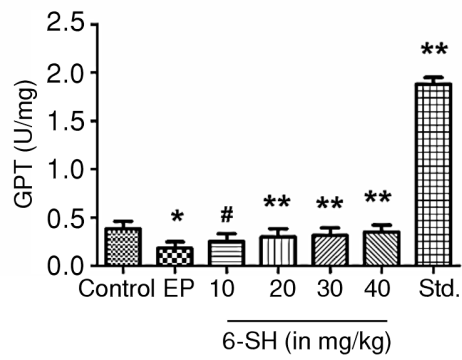

Figure 4. Effect of 6-SH on the levels of non-enzymatic antioxidants and enzymatic antioxidants in serum in edotoxin induced experimental periodontitis. ${ }^{*} \mathrm{p}<0.01$ vs. control, $\mathrm{p} p<0.05,{ }^{* *} \mathrm{p}<0.01$ vs. EP group

expressed in many tissues and body fluids in humans. Finally, acid phosphatase is a ubiquitous lysosomal enzyme that hydrolyzes organic phosphates under acidic $\mathrm{pH}$ conditions. The activities of these enzymes were significantly elevated in the EP rats, whereas the activities of all enzymes decreased in a dose-dependent manner in 6-SH-treated rats (Fig. 2).

Acute-phase proteins such as C-reactive protein and fibrinogen were activated in the EP rats (Fig. 3); however, the levels of these proteins were decreased in the treated group. 
Furthermore, we investigated the effects of 6$\mathrm{SH}$ on non-enzymatic antioxidant systems (e.g., ascorbic acid, $\alpha$-tocopherol, ceruloplasmin, and GSH) and antioxidant enzymes (e.g., CAT, SOD, GPx, and GST), which protect cells from the damaging effects of ROS. The levels of these antioxidants were significantly decreased in the EP rats, although ascorbic acid, GSH, showed considerably lower effects (Fig. 4). By contrast, the levels of these antioxidants were positively modulated in 6-SHtreated rats, while the standard-treated group exhibited an even greater increase.

In the histopathological examination, the control group showed normal rat maxillae architecture. Meanwhile, cellular infiltration and inflammatory cells were observed in the EP group. By contrast, in 6-SH-treated rats (30 and $40 \mathrm{mg} / \mathrm{kg}$ ), no such abnormal architecture was observed. This could indicate that the severity of PD was significantly controlled by the protective effects of 6-SH (Fig. 5).

\section{DISCUSSION}

Ginger has been used as a traditional medicinal herb in the Indian subcontinent and China for millennia (16), and its use eventually spread worldwide due to its immense medical significance $(12,13$, 15). Numerous studies have reported its antioxidant and anti-inflammatory actions via lipoxygenase activity inhibition. Ginger contains various bioactive compounds, among which gingerols are considered major bioactive components, and include 6,8 , and 10-gingerols based on the lengths of their unbranched alkyl chains. Of these, 6-gingerol (i.e., 6-shogaol; 1-4-hydroxy-3-methoxyphenyl-4-decen3 -one) is a major bioactive compound found in the dried rhizome of ginger and has various pharmacological actions including antioxidant, anti-inflammatory, analgesic-antipyretic, antibacterial, and antidiabetic activities $(12,15,17-20)$. In this study, 6-SH improved the condition of EP by acting on numerous mediators responsible for the progression of the disease. The destructive role of neutrophils in periodontal tissue rupture is well known based on the high levels of lysosomal enzymes and production of ROS (21-23). MPO exhibits proficient antimicrobial activity and has a critical role in tissue injury. Thus, people affected by PD show significant increases in MPO activity. ROS cause tissue damage in periodontitis due to the impairment of the antioxidant system, contributing to the inflammatory pathogenesis of PD (24-27). The results of this investigation revealed that $6-\mathrm{SH}$ treatment led to significant improvements in antioxidant activity, possibly because of its free radical-scavenging activity. Furthermore, the 6-SH-treated group showed significant improvements in the activities of the antioxidant enzymes CAT, SOD, GPx, and GST.
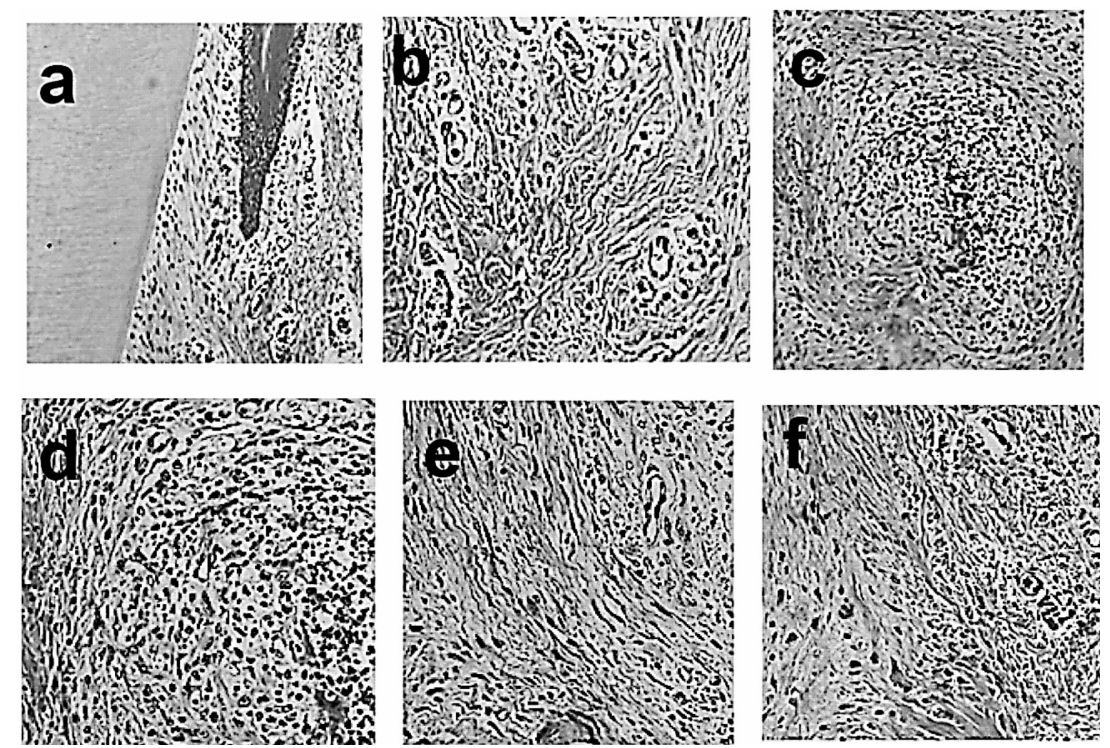

Figure 5. Histopathological examination of 6-SH on the tissues. a) Group 1: Control group showing normal architecture of gingival tissues; Group 2: EP group showing dense chronic inflammatory cells; Group 3: Dense collection of inflammatory cells (10 mg/kg); Group 4: Dense chronic inflammatory cells with plasma cells and lymphocytes $(20 \mathrm{mg} / \mathrm{kg}$ ); Group 5: Presence of diffuse chronic inflammatory cells (30 mg/kg); Group 6: Presence of diffuse chronic inflammatory cells ( $40 \mathrm{mg} / \mathrm{kg}$ ) 
The activity of 6-SH was also investigated on nonenzymatic antioxidants, the second line of antioxidant defense such as ascorbic acid, $\alpha$-tocopherol, ceruloplasmin, and GSH, which scavenge residual free radicals that escape decomposition by antioxidant enzymes. A marked improvement in the activities of these markers was observed in the 6$\mathrm{SH}$-treated group (Figure 4), in accordance with earlier studies (14, 28-31). Moreover, 6-SH prevents the destruction of lipids by limiting oxidative stress, resulting in a decrease in inflammation and the destruction of the periodontium. Cathepsin $\mathrm{D}$ has been reported as a key biomarker of PD and associated tissue destruction (32-35). In this investigation, 6-SH treatment caused a decrease in the levels of acidic lysosomal cysteine proteinases such as cathepsin B and cathepsin D, which have critical roles in intracellular proteolytic degradation processes. $\beta$-Glucuronidase and acid phosphatases act as key biomarkers of the destruction of non-collagenous components of the extracellular matrix and osteoclastic activity, respectively $(36,37)$. In this study, 6-SH treatment decreased the levels of these biomarkers, suggestive of its protective effect against periodontitis (Fig. 2). Various reports have suggested a role of excessive fibrinogen production in the upregulation of the immune response of the host, which is highly expressed in periodontitis infection and inflammation (38-40). In this study, fibrinogen expression was highly suppressed in 6-SH-treated rats compared to the EP group. The beneficial change in PD markers by 6-SH was further substantiated by the histopathological analyses.

\section{CONCLUSIONS}

The results of this investigation suggest a protective effect of 6-SH against EP via the regulation of key disease markers involved in the inflammatory process. Moreover, 6-SH improved antioxidant systems to counteract free radical-mediated damage. However, additional studies are warranted to clarify the detailed mechanism of its activity.

\section{Conflict of interest} declare.

The authors have no conflicts of interest to

\section{Funding}

This study was supported by Gansu Natural Science Foundation of China (No: 1610RJZA106).

\section{REFERENCES}

1. Aas J.A., Paster B.J., Stokes L.N., Olsen I., Dewhirst F.E.: J. Clin. Microbiol. 43, 5721 (2005).

2. Scannapieco F.A.: J. Periodontol. 70, 793 (1999).

3. Dzink J.L., Tanner A.C., Haffajee A.D., Socransky S.S.: J. Clin. Periodontol. 12, 648 (1985).

4. Hwang I.S., Hwang J.S., Hwang J.H., Choi H., Lee E. et al.: Curr. Microbiol. 66, 56 (2013).

5. Balunas M.J., Kinghorn A.D.: Life Sci. 78, 431 (2005)

6. Cragg G.M., Newman D.J.: Biochim. Biophys. Acta 1830, 3670 (2013).

7. Newman D.J., Cragg G.M., Snader K.M.: J. Nat. Prod. 66, 1022 (2003).

8. Newman D.J. Cragg G.M.: J. Nat. Prod. 75, 311 (2012).

9. Butler M.S.: J. Nat. Prod. 67, 2141 (2004).

10. Cragg G.M., Newman D.J., Snader K.M.: J. Nat. Prod. 60, 52 (1997).

11. Clark A.M.: Pharm. Res. 13, 1133 (1996).

12. Grzanna R., Lindmark L., Frondoza C.G.: J. Med. Food 8, 125 (2005).

13. Malhotra S., Singh A.P.: Nat. Prod. Rad. 2, 296 (2003).

14. Stoilova I., Krastanov A., Stoyanova A., Denev P., Gargova S.: Food Chem. 102, 764 (2007).

15. Afzal M., Al-Hadidi D., Menon M., Pesek J., Dhami M.S.: Drug Metab. Drug Interac. 18, 159 (2001).

16. Zadeh J.B., Kor N.M.: Euro. J. Exp. Bio. 4, 87 (2014).

17. Levy A.S.A., Simon O., Shelly J., Gardener M.: BMC Pharmacol. 6, 12 (2006).

18. Ling H., Yang H., Tan S.H., Chui W.K., Chew E.H.: Br. J. Pharmacol. 161, 1763 (2010).

19. Saptarini N.M., Sitorus E.Y., Levita J.: Int. J. Chem. 5, 12 (2013).

20. Patra J.K., Kim E.S., Oh K., Kim H-J., Kim Y. et al.: BMC Complement. Alternat. Med. 14, 343 (2014).

21. Scott D.A., Krauss J.: Front. Oral Biol. 15, 56 (2012)

22. Tobita M., Mizuno H.: Curr. Stem Cell Res. Ther. 5, 168 (2010).

23. Nussbaum G., Shapira L.: J. Clin. Periodontol. 38, 49 (2011)

24. Bandyopadhyay U., Das D., Banerjee R.K.: Current 77, 658 (1999).

25. Bergamini C.M., Gambetti S., Dondi A., Cervellati C.: Curr. Pharm. Des. 10, 1611 (2004) 
26. Chapple I.L.C., Matthews J.B.: Periodontol. 2000 43, 160 (2007).

27. Buonocore G., Perrone S., Tataranno M.L.: Semin. Fetal. Neonatal. Med. 15, 186 (2010).

28. Shanmugam K.R., Mallikarjuna K., Nishanth K., Kuo C.H., Reddy K.S.: Food Chem. 124, 1436 (2011).

29. Kikuzaki H., Nakatani N.: J. Food Sci. 58, 1407 (1993).

30. Sanwal S.K., Rai N., Singh J., Buragohain J.: Sci. Hort. 124, 280 (2010).

31. Jeena K., Liju V.B., Kuttan R.: Indian J. Physiol. Pharmacol. 57, 51 (2013).

32. Genco R.J., Borgnakke W.S.: Periodontol. 2000. 62, 59 (2013).

33. Taubman M. a., Valverde P., Han X., Kawai T.: J. Periodontol. 76, 2033 (2005).
34. Fusek M., Vetvicka V.: Czechoslovakia 149, 43 (2005).

35. Benes P., Vetvicka V., Fusek M.: Crit. Rev. Oncol. Hematol.68, 12 (2008).

36. Bou-Gharios G., Ponticos M., Rajkumar V., Abraham D.: Cell Prolif. 37, 207 (2004).

37. Bull H., Murray P.G., Thomas D., Fraser A.M., Nelson P.N.: Mol. Pathol.? 55, 65 (2002).

38. Rourke C., Curry N., Khan S. et al.: J. Thromb. Haemost 10, 1342 (2012).

39. Fort A., Borel C., Migliavacca E., Antonarakis S.E., Fish R.J. et al.: Blood 116, 2608 (2010).

40. Smiley S.T., King J.A., Hancock W.W.: J. Immunol. 167, 2887 (2001).

Received: 03.11.2017 